Document downloaded from:

http://hdl.handle.net/10251/60053

This paper must be cited as:

Remón Martín, L.; Arias, A.; Calatayud Calatayud, A.; Furlan, WD.; Monsoriu Serra, JA. (2012). Through-focus response of multifocal intraocular lenses evaluated with a spatial light modulator. Applied optics. 36(51):8594-8598. doi:10.1364/AO.51.008594.

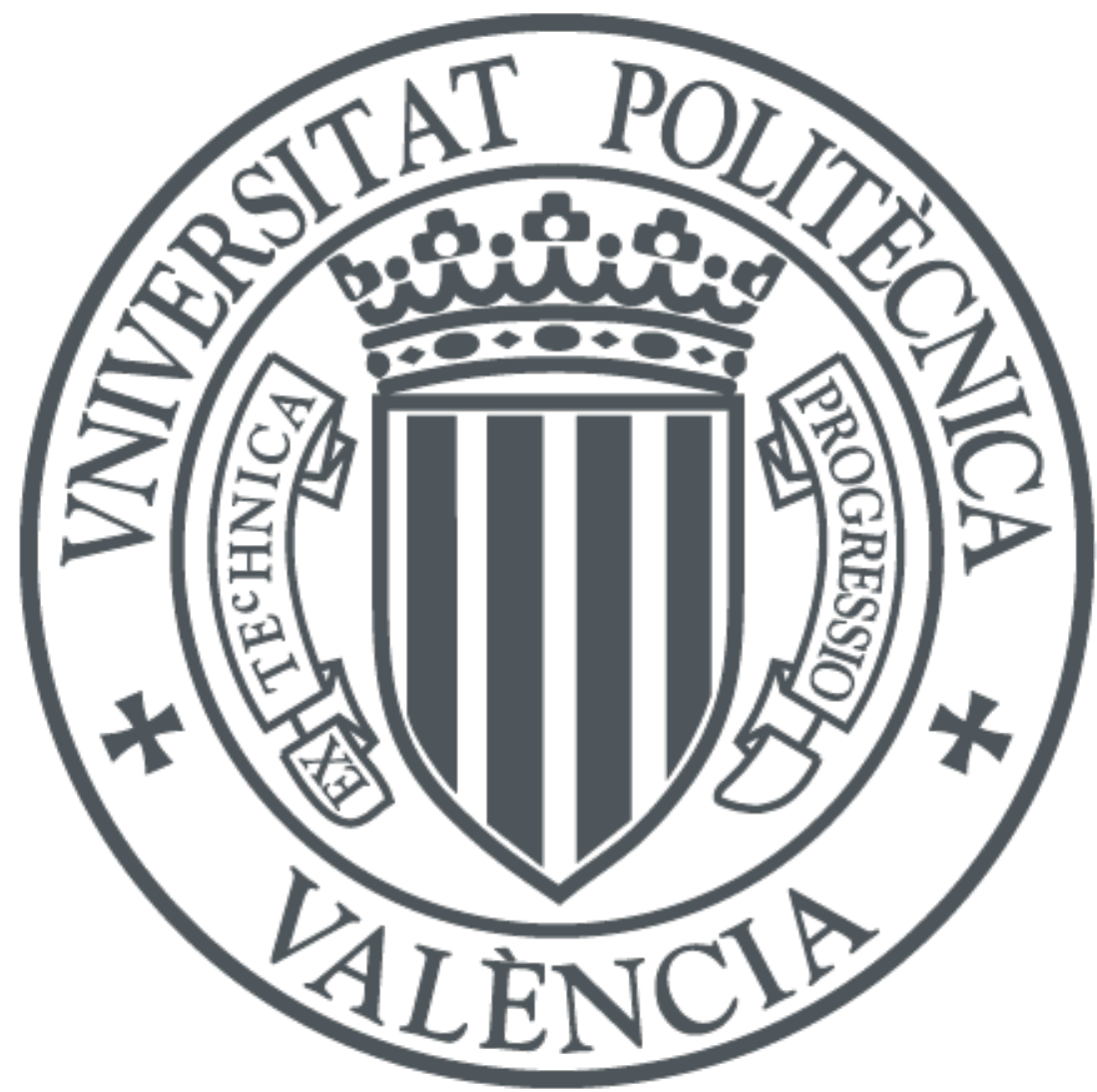

The final publication is available at

http://dx.doi.org/10.1364/AO.51.008594

Copyright Optical Society of America

Additional Information 


\title{
Through-focus response of multifocal intraocular lenses evaluated with a spatial light modulator
}

\author{
Laura Remón, ${ }^{1}$ Augusto Arias, ${ }^{2,3}$ Arnau Calatayud, 1 \\ Walter D. Furlan, ${ }^{3}$ and Juan A. Monsoriu, ${ }^{1^{*}}$ \\ ${ }^{1}$ Centro de Tecnologías Físicas, Universitat Politècnica de València, 46022 \\ Valencia, Spain \\ ${ }^{2}$ Centro de Investigación, Instituto Tecnológico Metropolitano A-365 \\ Medellín, Colombia \\ ${ }^{3}$ Departamento de Óptica, Universitat de València, 46100 Burjassot, Spain \\ *Corresponding author: jmonsori@fis.upv.es
}

\begin{abstract}
A new testing technique based on the use of a Liquid Crystal Spatial Light Modulator (SLM) is proposed to analyze the optical quality of multifocal intraocular lenses. Different vergences and decentrations of the incident beam can be programmed onto the SLM in order to record the Point Spread Function (PSF) for different object positions. From these axial PSFs, the through-focus Modulation Transfer Function (MTF) is computed. Because there are no moving parts in the experimental setup this method is fast and versatile to assess multifocal intraocular lenses. Experimental results confirm the potential of the proposed method. 2012 Optical Society of America
\end{abstract}

OCIS codes: (330.0330) Vision, color, and visual optics; (170.4470) Ophthalmology; (050.1970) Diffractive optics; (120.4800) Optical standards and testing.

\section{Introduction}

Although monofocal intraocular lenses are still frequently employed for treating cataracts, multifocal intraocular lenses (MIOLs) are nowadays an increasingly used modality that provides to users good vision, not only for far, but also for near objects. The performance of these lenses has been reported in numerous studies performed both in vivo and in vitro showing that decentration and tilt are two frequent and important factors affecting vision to be taken into account in cataract surgery (see For example [1-4] and the references therein). To assess the optical quality of MIOLs several experimental setups were designed in which the Point Spread Function (PSF) and/or the Modulation Transfer Function (MTF), measured for the distance and the near foci, are the essential merit functions. However, some valuable information, as for example the depth of focus (DOF), is missed if 
measurements are restricted to these two cases. To solve this problem, the Defocus Transfer Function was proposed as a theoretical tool for illustrating the OTF for all levels of defocus [5]. By calculating this function for a given spatial frequency, the simulated performance of implanted MIOLs on distance, intermediate, and near vision can be evaluated simultaneously. In order to properly sample the range of defocused planes between distance focus and near foci, different methods such as moving the detector (artificial retina) along the optical axis or generating different vergences in object space are possible. However in all these approaches the transition between the different defocused planes is attained by moving elements along the optical axis in the experimental setup. On the other hand the response of a MIOL to misalignments, such as tilt and decentration is a crucial issue for assessing its optical performance [6-8] and the tolerance to these factors is included in the battery of tests contained in the ISO 11979:2006 standard [9]. The influence of misalignments of monofocal intraocular lenses has been tested with different techniques such as using Purkinje images [10,11], Scheimpflug photography $[11,12]$ and ray tracing $[3,8,13]$. However, these techniques are not easily adapted to assess the performance of decentered MIOLs.

In this work, we describe a new objective technique for the assessment of MIOLs by means of the use of a Spatial Light Modulator (SLM). The programmable SLM is used to simulate different object vergences and pupil decentrations. The system provides the through-focus PSF of the lens under test in a totally automated procedure. We demonstrate how the axial PSF, with and without decentration, can be obtained with no moving parts in the experimental setup. This method is fast and versatile and it is presented as a powerful tool in the assessing of MIOLs because additionally to provide in a direct way the depth of focus of the lens under test other functions such as the through-focus MTF can be obtained. To show the capabilities of the proposed experimental setup, the performance and sensitivity of the apparatus is tested with a diffractive commercial MIOL.

\section{Experimental set up}

The optical system we propose for the measurement of the axial PSF of MIOLs is sketched in Fig.1. The most important features of the system relies on the liquid crystal on silicon Spatial Light Modulator (SLM) device (Holoeye PLUTO, 8-bit gray level, pixel pitch $\mathrm{d}=8 \mu \mathrm{m}$ and 1920x1080 pixels) which controls the illumination beam that impinges on the MIOL under test. The SLM is calibrated for a $2 \pi$ phase shift at $\lambda=633 \mathrm{~nm}$ (He-Ne laser). According to the data provided by the manufacturer, the SLM has for this wavelength an average reflectivity of about $60 \%$. The diffraction efficiency of the light reflected by the display is also limited by the fact that the fill factor (FF) of each elementary cell of the SLM lower than 1 . The calibration process 
includes a fundamental step conceived to compensate the wavefront distortions caused by the lack of flatness of the SLM and the other optical components. To this end we employed a Hartmann-Shack (HS) wavefront sensor (Thorlabs WFS 150-7 AR). First wavefront distortions are measured for zero modulation of the SLM. Then, the complex conjugated of the retrieved phase was addressed to the SLM to cancel the unwanted distorsions [14]. In addition, a linear carrier phase is modulated on the SLM in order to avoid the noise originated from the specular reflection (zero order of diffraction), and also the noise caused by high diffraction orders due to its pixelated structure. This linear carrier phase is compensated slightly tilting the SLM. In this way the addressed signal is guided into the first diffraction order at the object focal plane of the lens $\mathrm{L}_{2}$ in which a pin-hole acts as a spatial filter.

To simulate different object vergences and pupil decentrations on the MIOL the corresponding phase components were addressed to the SLM operating in phase-only modulation mode. The MIOL under tested is positioned in a wet cell with saline solution at the conjugated plane of the SLM. A lens tube microscope attached to a CCD camera is focused at the distance focus of the MIOL.

The phase on the SLM can be programmed to include several amounts of defocus and different values of decentrations within a pupil diameter. Mathematically the wrapped phase addressed to the SLM is expressed as:

$$
\phi_{S L M}(x, y)=\operatorname{circ}\left(\frac{\sqrt{\left(x-x_{c}\right)^{2}+y^{2}}}{R}\right)\left[\phi_{C}+\frac{D \pi}{\lambda}\left(\left(x-x_{c}\right)^{2}+y^{2}\right)\right] \text {, }
$$

where the function circ defines a binary circular aperture with radius $\mathrm{R}$, and $x_{c}$ is the amount of decentration along the $x$ axis. The term inside the brackets is the phase corresponding to different object vergences; $D$ is the defocus coefficient measured in diopters and $\lambda$ is the wavelength. $x_{C}$ is the linear carrier phase mentioned before that, acting as a prism, allows the separation of the signal from the noise. Figure 2 shows schematically the projected incident beam onto the MIOL under test. In our experimental setup the magnification between the SLM plane and the image plane $(z=0)$ is $M=f_{3} / f_{2}=0.5$ resulting as an effective pupil diameter of $3 \mathrm{~mm}$ at the MIOL plane. Fifty-two vergences in the range $-1.0 \mathrm{D}$ to $5.5 \mathrm{D}$ (in steps of $0.125 \mathrm{D}$ ) and three different values of decentrations: $0 \mathrm{~mm}, 0.25 \mathrm{~mm}$ and $0.5 \mathrm{~mm}$, were generated at the MIOL plane. The HS wavefront sensor was employed to verify the correctness of the induced vergences.

The different powers of the MIOL under test produce, in the primary focus planes, a focused component surrounded by the halo resulting from the other 
primary images. However, the intensity of these images differs in several orders of magnitude [15]. This would make accurate measurement of the PSF quite difficult unless dynamic range of the CCD would be enough to cover this difference. In our system (with a 12-bit gray-level CDD), we overcome this problem by recording, and then superimposing, 8 images with different exposure time, for each considered object vergence. i.e.; we synthesize a highdynamic range image PSF that includes the peak intensity of the in focus image and also the fainter image of the halo blur. From the experimental data, the MTF can be readily obtained by means of a Fast Fourier Transform algorithm.

The whole process (generation of different vergences on the SLM, the PSF image capture and data processing) is automated by means of dedicated software programmed in LabVIEW®. We want to emphasize, that with this method the values of the axial PSF, with and without decentrations, of a MIOL can be recorded with no moving parts in the experimental setup allowing the measurement in only fifty seconds.

\section{Results}

In order to test the capabilities of the proposed setup a commercial diffractive MIOL (Tecnis $®$ ZM900, developed by Advanced Medical Optics, Inc.) was tested. The lens is biconvex and made of a foldable silicone (polysiloxane). The posterior surface of the lens has a diffractive saw-tooth profile consisting of 32 rings with equal height $(h)$ that provides the optical power add of $\mathrm{P}_{\text {add }}\left(\lambda_{0}\right)=4.00 \mathrm{D}$ (3.2 D at the corneal plane) for the design wavelength $\left(\lambda_{0}=550 \mathrm{~nm}\right)$. As in our experiment we employed a different wavelength $(\lambda=633 \mathrm{~nm})$ two important effects should to be taken into account: The first one, is the change of the addition power [16] $\mathrm{P}_{\text {add }}(\lambda)=\left(\lambda / \lambda_{0}\right) \mathrm{P}_{\text {add }}\left(\lambda_{0}\right)=4.6 \mathrm{D}$. The second one is the change in the diffraction efficiency $(\eta)$ for the distance and near foci. This parameter is defined as [17]:

$$
\eta_{m}=\sin c^{2}(\alpha-m)
$$

where, in our case, $m=0$ for the distance focus and $m=1$ for the near focus. The parameter $\alpha$ is the maximum phase shift in units of wavelengths: $\alpha=\left(n_{a}-n_{i o l}\right)$ $h / \lambda$, where $n_{a}$ and $n_{\text {iol }}$ are the refraction indexes of the aqueous humor and the IOL, respectively, and $h$ is the height of the diffractive saw-tooth profile. In the considered lens, $\alpha=0.5$ for design wavelength $\lambda_{0}$ and thus only in this case the distance and near foci are equi-energetic. For wavelengths other than the design one the diffraction efficiency can be calculated as: $\alpha(\lambda)=\left(\lambda_{0} / \lambda\right) \alpha\left(\lambda_{0}\right)$. Thus, in our experiment, the expected ratio between the near focus intensity and distance focus intensity is $\alpha=0.61$. 
Figure 3 shows the experimental results for the axial PSFs given by the MIOL under test, where the different object vergences were obtained by changing the illuminating beam with the SLM. In the same figure, the numerical result computed using the Fresnel-Kirchhoff approximation is shown for comparison. As can be seen, the experimental results are in very good agreement with the theoretical predictions.

The PSF of the lens for different object positions and lens decentrations is shown in Fig. 4. The arrows indicate the lateral displacements of the center of mass of the PSF with respect to the centered in focus image. For each decentration, the near focus (4.6 D) provided by the IOL is well defined but it is laterally shifted. The PSFs for intermediate vergences, between distance and near, is extended in a much larger area and in all decentered images a comalike aberration is noticeable. The displacement of the focal spot vs. object vergence was estimated by calculating the center of mass of the PSFs (the result is plotted in Fig. 5). As expected according to the Prentice law, a nearly linear variation of the PSF center of mass between the near and distance foci can be observed. The lateral shift is proportional to the pupil decentration.

From the composed experimental axial PSFs we compute the values of MTF as a function of defocus for centered and decentered pupils. The result is shown in Fig. 6. The horizontal lines in this figure represent objects at distance and near planes. The profiles along these two lines provide the classical representation of the MTF which are shown in Fig. 7. Additionally in the same figure the MTFs corresponding to decentered pupils are also shown. Note that, there is a non-linear dependence of the MTF on the decentration, being of little influence for small decentrations $\left(x_{\mathrm{c}} \leq 0,25 \mathrm{~mm}\right)$. In fact the MTF for small values of decentration indicates a little difference compared with the centered case. However for $x_{\mathrm{c}}=0,50 \mathrm{~mm}$ the induced off-axis aberrations produce a noticeable lower values of the MTF at both foci.

\section{Conclusions}

In this work, a new method to obtain the axial PSF generated by MIOLs is proposed. This merit function is recorded for different object vergences which are generated creating a divergent beam of light (with variable degree of divergence) by changing the phase of the incoming light by means of a SLM, and consequently, with no moving parts in the experimental setup. Moreover, this device allows obtain the MIOL response to a variable amount of decentration and tilt. The whole process is performed automatically in a few seconds. From the experimental PFS, the through-focus MTF is readily obtained. To test our proposal, a commercial diffractive MIOL has been analyzed by computing these merit functions. The experimental results are in good agreement with the lens specifications provided by the manufacturer. 
The versatility provided by the SLM can be utilized to simulate the effect that other high order aberrations produce on the MIOL optical quality. Finally it is worth to mention that the experimental analysis of chromatic properties of MIOLs $[18,19]$ is readily possible with our proposal simply by changing the light source.

Acknowledgements: We acknowledge the financial support from Ministerio de Ciencia e Innovación (grants FIS2011-23175 and TRA2009-0215), Generalitat Valenciana (grant PROMETEO2009-077), and Universitat Politècnica de València (PAID-05-11), Spain. L.R. acknowledges a fellowship of "Fundación Cajamurcia", Spain. 


\section{References and notes}

1. V. Portney, "Optical testing and inspection methodology for modern intraocular lens," J. Cataract. Refract. Surg. 18, 607-613 (1992).

2. R. Rawer, W. Stork, C. W. Spraul, and C. Lingenfelder, "Imaging quality of intraocular lenses," J. Cataract. Refract. Surg. 31, 1618-1631 (2005).

3. S. Barbero, S. Marcos, and I. Jiménez-Alfaro, "Optical aberrations of intraocular lenses measured in vivo and in vitro," J. Opt. Soc. Am. A 20, 1841-1851 (2003).

4. J. Schwiegerling and E. DeHoog, "Problems testing diffractive intraocular lenses with Shack-Hartmann sensors," Appl. Opt. 49, D62-D68 (2010).

5 Schwiegerling, "Analysis of the optical performance of presbyopia treatments with the defocus transfer function," J. Refract. Surg. 23, 965-971 (2007).

6. D. A. Atchison, "Optical design of intraocular lenses. III. On-axis performance in the presence of lens displacement, " Optom. Vis. Sci. 66, 671681 (1989).

7. F. Taketani, T. Matuura, E. Yukawa, and Y. Hara, "Influence of intraocular lens tilt and decentration on wavefront aberrations," J. Cataract. Refract. Surg. 30, 2158-2162 (2004).

8. G. E. Altmann, L. D. Nichamin, S. S. Lane, and J. S. Pepose, "Optical performance of 3 intraocular lens designs in the presence of decentration," J. Cataract. Refract. Surg. 31, 574-585 (2005).

9. ISO/FDIS 11979-9 Ophthalmic implants - Intraocular lenses - Part 9: Multifocal intraocular lenses (2006).

10. P. Phillips, J. Pérez-Emmanuelli, H. D. Rosskothen, and C. J. Koester, "Measurement of intraocular lens decentration and tilt in vivo," J. Cataract. Refract. Surg. 14, 129-135 (1988).

11. A. de Castro, P. Rosales, and S. Marcos, "Tilt and decentration of intraocular lenses in vivo from Purkinje and Scheimpflug imaging," J. Cataract. Refract. Surg. 33, 418-429 (2007).

12. K. Sasaki, Y. Sakamoto, T. Shibata, H. Nakaizumi, and Y. Emori, "Measurement of postoperative intraocular lens tilting and decentration using Scheimpflug images," J. Cataract. Refract. Surg. 15, 454-457 (1989).

13. T. Eppig, K. Scholz, A. Löffler, A. Mebner, and A. Langenbucher, "Effect of decentration and tilt on the image quality of aspheric intraocular lens designs in a model eye," J. Cataract. Refract. Surg. 35, 1091-1100 (2009).

14. A. Calatayud, J. A. Rodrigo, L. Remón, W. D. Furlan, G. Cristóbal, and J. A. Monsoriu, "Experimental generation and characterization of Devil's vortexlenses," Appl. Phys. B. 106, 915-919 (2012).

15. M. J. Simpson, "Diffractive multifocal intraocular lens image quality," App. Opt. 31, 3621-3626 (1992).

16. D. Atchison, M.Ye, A. Bradley, M.J.Collins.; X. Zhang; H.A. Rahman and L.N. Thibos. "Chromatic aberration and optical power of a diffractive bifocal contact len,". Optom. Vis. Sc,. 69, 797-804 (1992). 
17. V. Portney, "Light distribution in diffractive multifocal optics and its optimization," J. Cataract. Refract. Surg. 37, 2053-2059 (2011).

18. J. Schwiegerling and J. Choi, "Application of the polychromatic defocus transfer function to multifocal lenses," J. Refract. Surg. 24, 965-969 (2008).

19. F. Castignoles, M. Flury, and T. Lepine, "Comparison of the efficiency, MTF and chromatic properties of four diffractive bifocal intraocular lens designs," Opt. Express 18, 5245-5256 (2010). 


\section{Figure captions}

FIGURE 1. Experimental setup for obtaining the axial PSF of a MIOL. The SLM (Holoeye PLUTO, 8-bit gray-level, pixel size $d=8 \mu \mathrm{m}$ resolution 1920 x1080 pixels) modulates a collimated laser at $\lambda=633 \mathrm{~nm} . \mathrm{L}_{2}\left(f_{2}=20 \mathrm{~cm}\right)$ and $\mathrm{L}_{3}\left(f_{3}=10 \mathrm{~cm}\right)$ conform an afocal relay system. A 10x Zeiss PlanApochromat objective is attached to the CCD camera (12-bit gray-level, pixel pitch of $3,75 \mu \mathrm{m}$ and $1280 \times 960$ pixels) to record the PSF images.

FIGURE 2. Effect of a decentered illumination respect to the IOL position. The shift amount in the $\mathrm{x}$-axis is given by $\mathrm{x}_{\mathrm{c}}$.

FIGURE 3. Experimental and theoretical axial intensity produced by the MIOL under test with no decentration.

FIGURE 4. Experimental PSFs, for different object vergences and three different decentrations: $x c$. a) $0 \mathrm{~mm}$, b) $0.25 \mathrm{~mm}$ and c) $0.50 \mathrm{~mm}$.

FIGURE 5. Lateral shift of the PSFs center of mass as a function of the vergence for the three decentration values.

FIGURE 6. Axial MTF computed from the experimental PSFs for different amount of decentration: a) $0 \mathrm{~mm}$, b) $0.25 \mathrm{~mm}$ and c) $0.50 \mathrm{~mm}$.

FIGURE 7. MTFs for the in focus images a) distance focus and b) near focus, obtained from Fig. 6 (horizontal lines). 
Figure 1

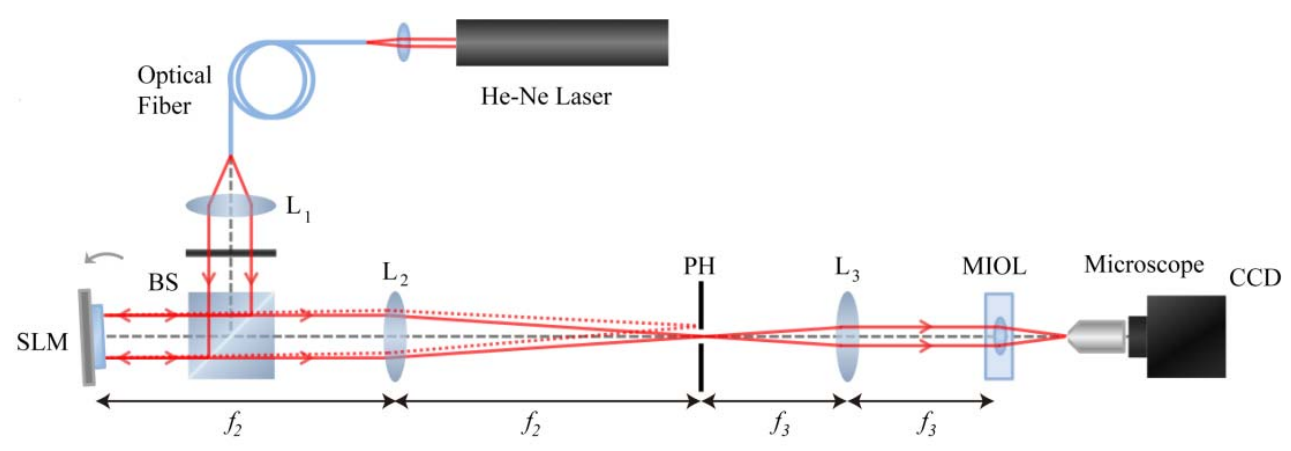


Figure 2

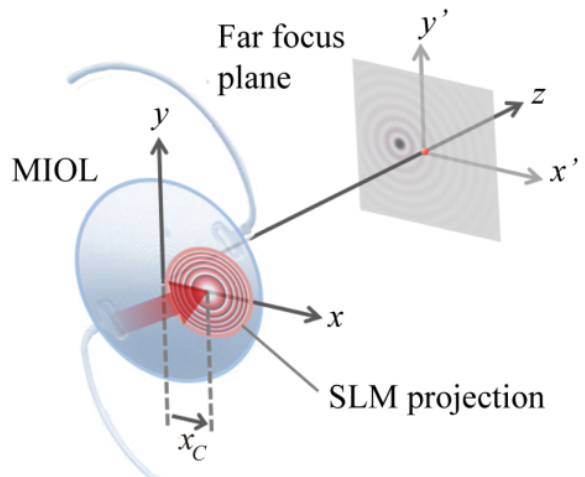


Figure 3

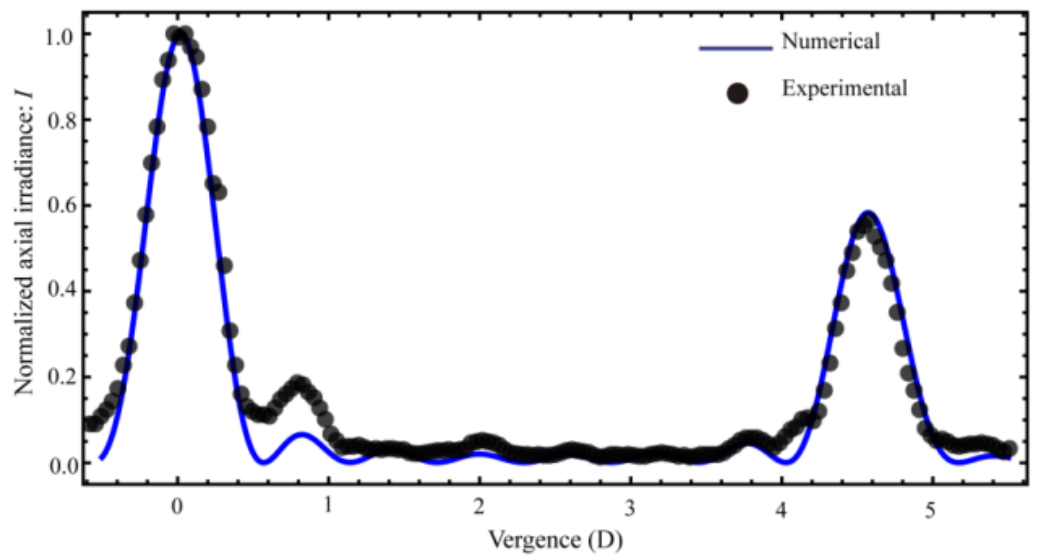


Figure 4

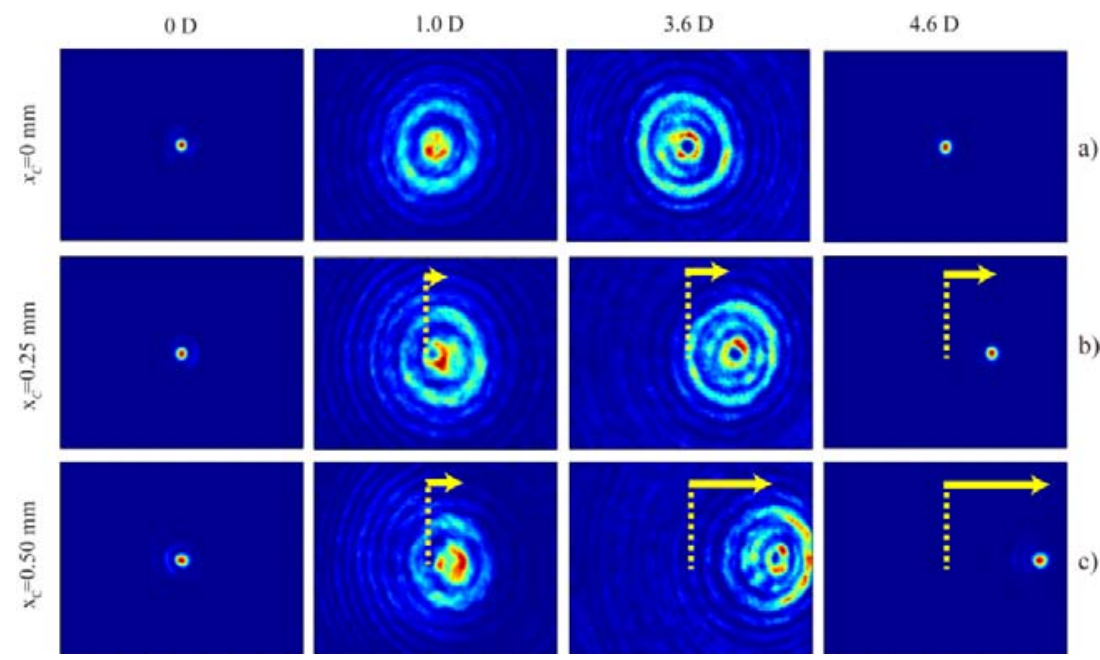


Figure 5

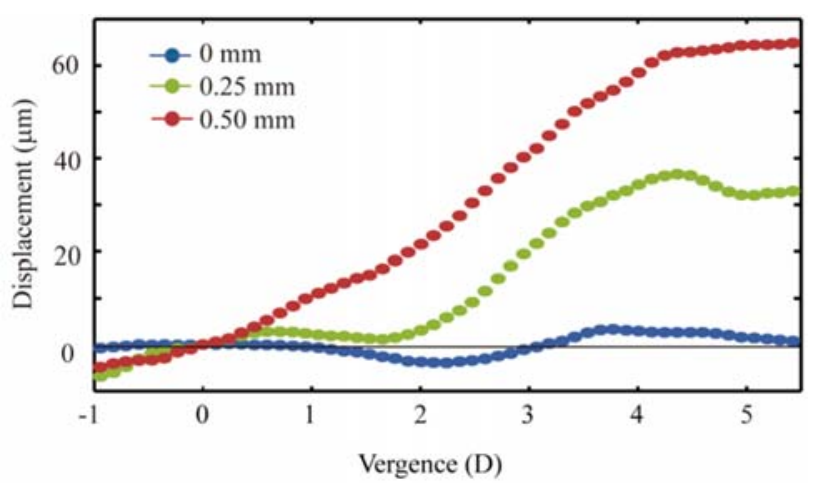


Figure 6
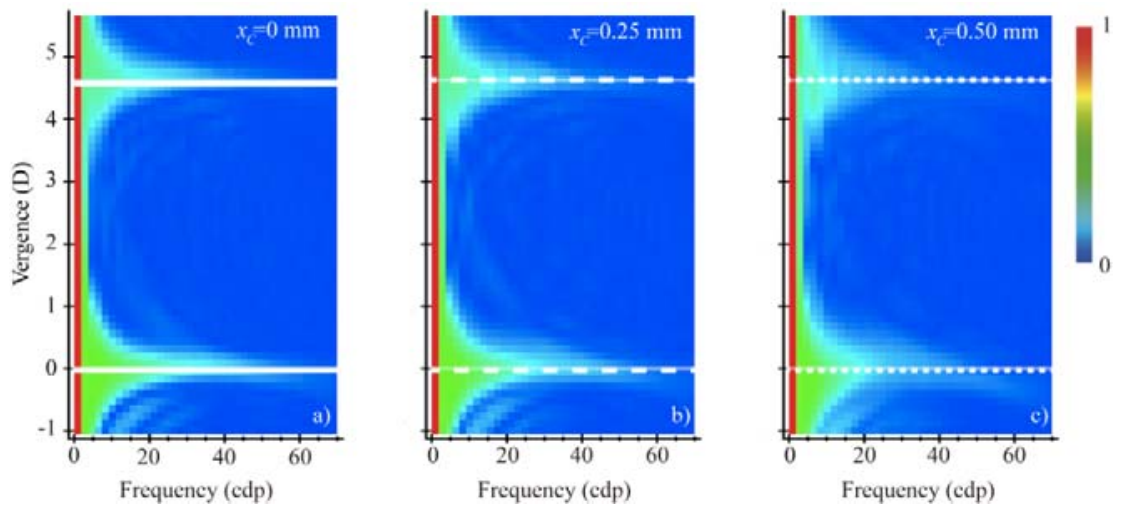
Figure 7
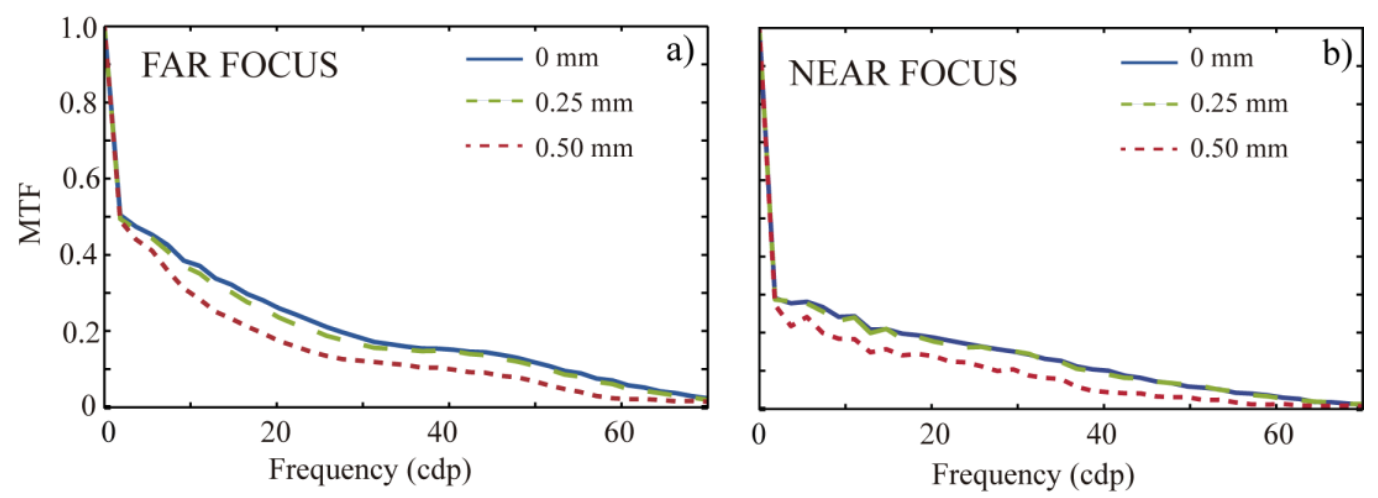